\title{
PERTURBATION CLASSES FOR SEMI-FREDHOLM OPERATORS ON SUBPROJECTIVE AND SUPERPROJECTIVE SPACES
}

\author{
Manuel González, Antonio Martínez-Abejón and Margot Salas-Brown
}

\author{
Universidad de Cantabria, Facultad de Ciencias, Departamento de Matemáticas \\ E-39071 Santander, España; gonzalem@unican.es \\ Universidad de Oviedo, Facultad de Ciencias, Departamento de Matemáticas \\ E-33007 Oviedo, España; ama@uniovi.es \\ Universidad de Oriente, Departamento de Matemáticas \\ 6101 Cumaná, Venezuela; msalas@sucre.udo.edu.ve
}

\begin{abstract}
We prove that the component $P \Phi_{+}(X, Y)$ of the perturbation class for the upper semi-Fredholm operators between Banach spaces $X$ and $Y$ coincide with the strictly singular operators when every closed infinite dimensional subspace of $X$ contains an infinite dimensional complemented subspace whose complement is isomorphic to $X$. Similarly, we prove that the component $P \Phi_{-}(X, Y)$ of the perturbation class for the lower semi-Fredholm operators coincide with the strictly cosingular operators when every infinite codimensional subspace of $Y$ is contained in an infinite codimensional complemented subspace isomorphic to $Y$. We also give examples of Banach spaces satisfying the aforementioned conditions.
\end{abstract}

\section{Introduction}

The perturbation classes problem arises in the study of the stability of Fredholm and semi-Fredholm operators under additive perturbations. Let $\mathscr{L}(X, Y)$ denote the (continuous linear) operators between the Banach spaces $X$ and $Y$. An operator $T \in \mathscr{L}(X, Y)$ is said to be upper semi-Fredholm $\left(T \in \Phi_{+}\right)$if its kernel $N(T)$ is finite dimensional and its range $R(T)$ is closed; $T$ is said to be lower semi-Fredholm $\left(T \in \Phi_{-}\right)$if its range is closed and finite codimensional, and $T$ is said to be Fredholm $(T \in \Phi)$ if it is both upper and lower semi-Fredholm. Let $\mathscr{A}$ be any of the classes $\Phi_{+}, \Phi_{-}$or $\Phi$. The perturbation class of $\mathscr{A}$ is defined by its components in $\mathscr{L}(X, Y)$, when $\mathscr{A}(X, Y)$ is non-empty:

$$
P \mathscr{A}(X, Y):=\{K \in \mathscr{L}(X, Y): K+T \in \mathscr{A}(X, Y) \text { for all } T \in \mathscr{A}(X, Y)\} .
$$

The components $P \mathscr{A}(X, Y)$ were studied in [17] in the case $X=Y$ and in [2] in the general case. It was proved in [22] that $P \Phi$ coincides with the inessential operators $\mathscr{I} n$ when it is defined, but the perturbation classes $P \Phi_{+}$and $P \Phi_{-}$have been identified only in a few cases. Kato showed that the strictly singular operators $\mathscr{S} \mathscr{S}$ are contained in $P \Phi_{+}[16$, Theorem 5.2], Vladimirskii proved that the strictly

doi:10.5186/aasfm.2011.3625

2010 Mathematics Subject Classification: Primary 47A53, 47A55.

Key words: Perturbation class, semi-Fredholm operator, strictly singular operator, strictly cosingular operator, subprojective space, superprojective space.

Supported in part by MICINN (Spain), Grant MTM2010-20190 and CI, UDO (Venezuela), Grant N CI-2-010301-1675-10. 
cosingular operators $\mathscr{S} \mathscr{C}$ are contained in $P \Phi_{-}[24$, Corollary 1], and it is a consequence of the continuity of the index for semi-Fredholm operators that both $P \Phi_{+}$ and $P \Phi_{-}$are contained in $\mathscr{I} n$ (see [5, Theorem 5.6.9]).

Recall that an operator $T: X \longrightarrow Y$ is in $\mathscr{S} \mathscr{S}$ if its restriction $\left.T\right|_{E}$ is an isomorphism for no infinite dimensional subspace $E$; $T \in \mathscr{S} \mathscr{C}$ if $Q_{F} T$ is surjective for no infinite codimensional closed subspace $F$ of $Y$, where $Q_{F}: Y \longrightarrow Y / F$ is the quotient operator, and $T \in \mathscr{I} n$ if for every $A \in \mathscr{L}(Y, X), I_{X}-A T \in \Phi$.

The perturbation classes problem asks whether $\mathscr{S} \mathscr{S}$ and $\mathscr{S} \mathscr{C}$ coincide with $P \Phi_{+}$ and $P \Phi_{-}$respectively. This problem was formulated by Gohberg, Markus and Feldman [11, p. 74]) for the upper semi-Fredholm operators. Later, it was explicitly stated in [5, page 101], [22, 26.6.12], [23, Section 3] and [3]. Finally, it was proved in [12] that there exists a complex separable Banach space $Z$ for which $P \Phi_{+}(Z) \neq \mathscr{S} \mathscr{S}(Z)$ and $P \Phi_{-}\left(Z^{*}\right) \neq \mathscr{S} \mathscr{C}\left(Z^{*}\right)$. However, there is still interest in finding spaces $X$ and $Y$ for which $P \Phi_{+}(X, Y)=\mathscr{S} \mathscr{S}(X, Y)$ or $P \Phi_{-}(X, Y)=\mathscr{S} \mathscr{C}(X, Y)$ because these results provide intrinsic characterizations of the operators $K$ in the respective classes; i.e., characterizations involving the action of $K$ instead of the properties of the sums of $K$ with all the operators in $\Phi_{+}(X, Y)$ or $\Phi_{-}(X, Y)$. Moreover, the aforementioned space $Z$ of [12] is certainly special: it is a finite product of hereditarily indecomposable spaces. The existence of hereditarily indecomposable Banach spaces was only recently proved in [15]. So the perturbation classes problem still remains open for many classical Banach spaces.

Provided $\Phi_{+}(X, Y) \neq \emptyset$, we have $P \Phi_{+}(X, Y)=\mathscr{S} \mathscr{S}(X, Y)$ in the following cases:

(1) $Y$ subprojective $[17,1]$;

(2) $X=Y=L_{p}(\mu), 1 \leq p \leq \infty[25]$;

(3) $X$ hereditarily indecomposable [1, Theorem 3.14];

(4) $X$ is separable and $Y$ contains a complemented copy of $C[0,1][3]$;

(5) $X=L_{p}(0,1)$ when $1<p<2$ and $Y$ satisfies the Orlicz property [14];

(6) $X=L_{1}(0,1)$ and $Y$ is weakly sequentially complete [14];

(7) $X=L_{p}(0,1)$ with $2 \leq p \leq \infty[14]$.

Also, provided $\Phi_{-}(X, Y) \neq \emptyset$, we have $P \Phi_{-}(X, Y)=\mathscr{S} \mathscr{C}(X, Y)$ in the following cases:

(1') $X$ superprojective $[17,1]$;

(2') $X=Y=L_{p}(\mu), 1 \leq p \leq \infty[25]$;

(3') $Y$ quotient indecomposable [1, Theorem 3.14];

(4') $X$ contains a complemented copy of $\ell_{1}$ and $Y$ is separable [3];

(5') $Y=L_{p}(0,1)$ when $2<p<\infty$ and $X^{*}$ satisfies the Orlicz property [14];

(6') $Y=L_{p}(0,1)$ with $1 \leq p \leq 2[14]$.

In this paper, we introduce the notions of strongly subprojective and strongly superprojective Banach space, which strengthen those of subprojective and superprojective Banach space introduced in [26]. We remark that all known examples of subprojective spaces and superprojective spaces are respectively strongly subprojective and strongly superprojective. Next, we prove that if $X$ is strongly superprojective, then $P \Phi_{+}(X, Y)=\mathscr{S} \mathscr{S}(X, Y)$ for all spaces $Y$ (Theorem 2.6), and if $Y$ is strongly superprojective, then $P \Phi_{-}(X, Y)=\mathscr{S} \mathscr{C}(X, Y)$ for all spaces $X$ (Theorem 3.7).

We point out that although Theorem 3.7 is a certain dual form of Theorem 2.6, its proof does not follow by duality from it. This is because, given $T \in \mathscr{L}(X, Y)$, 
the implications $T^{*} \in \mathscr{S} \mathscr{S} \Rightarrow T \in \mathscr{S} \mathscr{C}$ and $T^{*} \in \mathscr{S} \mathscr{C} \Rightarrow T \in \mathscr{S} \mathscr{S}$ hold but their converses fail. See [21, Examples 1 and 2]. Moreover, the proof of Theorem 3.7 is technically more complicated than that of Theorem 2.6 because the former one involves quotients instead of subspaces.

\section{Operators on strongly subprojective spaces}

A Banach space $X$ is said to be subprojective if every infinite dimensional closed subspace $M$ of $X$ contains an infinite dimensional subspace $N$ complemented in $X$. Clearly, a closed subspace of a subprojective space is also subprojective. This concept was introduced by Whitley [26]. Here we consider a strengthening of it.

Definition 2.1. A Banach space $X$ is said to be strongly subprojective if every infinite dimensional closed subspace $M$ of $X$ contains an infinite dimensional subspace $N$ complemented in $X$ with complement isomorphic to $X$.

The following remark will allow us to show that all the known examples of subprojective spaces (see Proposition 2.4) are strongly subprojective.

Remark 2.2. If the subspace $N$ in the definition of subprojective space can be taken isomorphic to its square $(N \simeq N \times N)$ then $X$ is strongly subprojective.

Proof. Let $M$ be an infinite dimensional closed subspace of a subprojective space $X$. Then there exist closed subspaces $N$ and $H$ of $X$ such that $X=N \oplus H$ and $N \subset M$. By hypothesis, $N$ contains two closed subspaces $N_{1}$ and $N_{2}$ such that $N \simeq N_{1} \simeq N_{2}$ and $N=N_{1} \oplus N_{2}$. Therefore, $N_{1}$ is a subspace of $M, N_{2} \oplus H \simeq X$ and

$$
X=N_{1} \oplus\left(N_{2} \oplus H\right),
$$

which proves that $X$ is strongly subprojective.

Remark 2.3. Recall that a compact space $K$ is said to be scattered (or dispersed) if every non-empty subset of $K$ has an isolated point. As examples, we mention:

(1) Let $\kappa$ be any ordinal. The interval $[0, \kappa]=\{\alpha$ ordinal $: 0 \leq \alpha \leq \kappa\}$, endowed with the order topology, is a scattered compact.

(2) Let $\Gamma$ be a set, endowed with the discrete topology. The one-point compactification $\Gamma_{\infty}$ is a scattered compact and $C\left(\Gamma_{\infty}\right)$ is isomorphic to $c_{0}(\Gamma)$.

Note that $\ell_{p} \simeq \ell_{p} \times \ell_{p}$ for $1 \leq p<\infty$ and $c_{0} \simeq c_{0} \times c_{0}$. Therefore, Remark 2.2 can be applied to obtain the following result.

Proposition 2.4. The following Banach spaces are strongly subprojective:

(1) The sequence spaces $\ell_{p}$ for $1 \leq p<\infty$ and $c_{0}$.

(2) The James space $J$.

(3) The Lorentz sequence spaces $d(w, p)$ for $1 \leq p<\infty$ and $w=\left(w_{n}\right)$ a nonincreasing null sequence with $\sum_{n=1}^{\infty} w_{n}$ divergent. This applies to $\ell_{p, q}$ for $1 \leq p, q<\infty$.

(4) The Baernstein spaces $B_{p}$ for $1<p<\infty$.

(5) The Tsirelson space $T$.

(6) The function spaces $L_{p}(0,1)$ for $2 \leq p<\infty$.

(7) The function spaces $L_{p}(0, \infty) \cap L_{2}(0, \infty)$ for $1 \leq p \leq 2$.

(8) The Lorentz spaces $\Lambda_{W, p}(0,1), L_{p, q}(0, \infty)$ and $\bar{L}_{p, q}(0,1)$ for $2<p<\infty$ and $1 \leq q<\infty$. 
(9) The spaces of continuous functions $C(K)$, with $K$ a scattered compact.

(10) Closed subspaces of the previous examples.

Proof. (1) Denoting by $X$ any of these spaces, every infinite dimensional closed subspace of $X$ contains a subspace isomorphic to $X$ and complemented in $X[4$, Proposition 2.2.1].

(2) Every infinite dimensional closed subspace of $J$ contains a subspace isomorphic to $\ell_{2}$ and complemented in $J$ [9, Corollary 2.d.4].

(3) Every infinite dimensional closed subspace of $d(w, p)$ contains a subspace isomorphic to $\ell_{p}$ and complemented in $d(w, p)$ [18, Proposition 4.e.3].

(4) Every infinite dimensional closed subspace of $B_{p}$ contains a subspace isomorphic to $\ell_{p}$ and complemented in $B_{p}$ [7, Theorem 0.15].

(5) Let $\left\{t_{n}\right\}$ denote the unit basis of $T$. By [7, Proposition II.7], every closed subspace of $T$ contains a subspace $N$ complemented in $T$ and isomorphic to the closed subspace generated by a subsequence of the basis $\left\{t_{n}\right\}$. Moreover, [7, Proposition I.12] ensures that $N \simeq N \times N$.

(6) Every infinite dimensional closed subspace of $L_{p}(0,1)$ is either isomorphic to $\ell_{2}$ and complemented, or contains a subspace isomorphic to $\ell_{p}$ and complemented in $L_{p}(0,1)$ [4, Corollary 6.4.9].

(7) The argument given in (6) applies in this case [8, Theorem 4.1].

(8) The argument given in (6) applies for $\Lambda_{W, p}(0,1)$ and $L_{p, q}(0, \infty)$. See [10, Remark 5.7] and [6, Theorem 2.5]. For $L_{p, q}(0,1)$, the result follows from (10), since $L_{p, q}(0,1)$ is a closed subspace of $L_{p, q}(0, \infty)$.

(9) Every infinite dimensional closed subspace of $C(K)$ contains a subspace isomorphic to $c_{0}$ and complemented in $C(K)$ [19, Theorem 11].

(10) Given a pair of closed subspaces $M$ and $Z$ of $X$ with $M \subset Z$, if $M$ is complemented in $X$, then it is also complemented in $Z$.

The next result will be useful later.

Proposition 2.5. Let $X$ be a strongly subprojective Banach space. Then every finite codimensional closed subspace of $X$ contains a subspace isomorphic to $X$. Consequently, $\Phi_{+}(X, Y)$ is non-empty if and only if $Y$ contains a subspace isomorphic to $X$.

Proof. Let $Z$ be a closed subspace of $X$ with $\operatorname{dim} X / Z=n$. Since $X$ is strongly subprojective, $X$ contains an infinite codimensional subspace $X_{0}$ isomorphic to $X$. Let $Z_{0}$ be a closed $n$-codimensional subspace of $X$ containing $X_{0}$. Since $Z$ and $Z_{0}$ are isomorphic, the first assertion is clear.

For the second assertion, note that $\Phi_{+}(X, Y)$ is non-empty if and only if $Y$ contains a closed subspace isomorphic to a finite codimensional subspace of $X$.

Let us give the main result of this section.

Theorem 2.6. Let $X$ be a strongly subprojective space and let $Y$ be a Banach space. If $\Phi_{+}(X, Y) \neq \emptyset$ then $P \Phi_{+}(X, Y)=\mathscr{S} \mathscr{S}(X, Y)$.

Proof. It is enough to show that, given $K \in \mathscr{L}(X, Y) \backslash \mathscr{S} \mathscr{S}(X, Y)$, there exists $T \in \Phi_{+}(X, Y)$ such that $T+K \notin \Phi_{+}$.

Since $K$ is not strictly singular, there exists an infinite dimensional closed subspace $V$ of $X$ such that $\left.K\right|_{V}$ is an isomorphism; hence $\left.K\right|_{V} \in \Phi_{+}(V, Y)$. As $X$ is 
strongly subprojective, we may assume that

$$
X=V \oplus X_{1} \text { with } X_{1} \simeq X .
$$

By Proposition 2.5, $Y$ has a closed subspace $L$ isomorphic to $X$. Taking into consideration the relative positions of the subspaces $K(V)$ and $L$ inside $Y$, three cases may happen:

(a) $K(V) \cap L$ finite dimensional and $K(V)+L$ closed;

(b) $K(V) \cap L$ is infinite dimensional;

(c) $K(V) \cap L$ finite dimensional and $K(V)+L$ not closed.

(a) As $L$ is strongly subprojective, by Proposition 2.5, the closed complement of $K(V) \cap L$ in $L$ contains a subspace isomorphic to $L$. Thus we can assume $K(V) \cap L=$ $\{0\}$.

Let $S: X_{1} \longrightarrow L$ be a bijective isomorphism. We consider the operator

$$
T: X=V \oplus X_{1} \longrightarrow K(V) \oplus L \subset Y
$$

that maps $v+x_{1}$ to $-K(v)+S\left(x_{1}\right)$, where $v \in V$ and $x_{1} \in X_{1}$. Clearly $T \in \Phi_{+}$. However $\left.(T+K)\right|_{V}=0$, so $T+K \notin \Phi_{+}$, and we are done.

(b) Assume $K(V) \cap L$ is infinite dimensional. Since $L$ is strongly subprojective, there exists a closed subspace $W$ contained in $V$ and a closed subspace $L_{3}$ in $L$ such that $L_{1}:=K(W) \cap L$ is infinite dimensional, $L_{3}$ is isomorphic to $L$ and $L=L_{1} \oplus L_{3}$. Let $V_{1}:=\left(\left.K\right|_{V}\right)^{-1}\left(L_{1}\right)$. By the strong subprojectivity of $X$, there exist an infinite dimensional closed subspace $V_{2}$ of $V_{1}$ and a closed subspace $X_{2}$ of $X$ such that $X_{2}$ is isomorphic to $X$ and $X=V_{2} \oplus X_{2}$. Since $\left.K\right|_{V_{2}}$ is an isomorphism and $K\left(V_{2}\right)+L_{3}$ is closed, we are in the conditions of case (a).

(c) As in case (a), we can assume that $K(V) \cap L=\{0\}$ and $K(V)+L$ not closed. In order to prove that $K \notin P \Phi_{+}(X, Y)$, it is enough to find a compact operator $K_{1} \in \mathscr{L}(X, Y)$ so that $\operatorname{dim}\left(K+K_{1}\right)(V) \cap L=\infty$; indeed, once the operator $K_{1}$ has been found, since $\left.\left(K+K_{1}\right)\right|_{V} \in \Phi_{+}(V, Y)$, the operator $K+K_{1}$ satisfies the conditions of case (b), which leads to $K+K_{1} \notin P \Phi_{+}(X, Y)$, and therefore $K \notin P \Phi_{+}(X, Y)$.

In order to find that operator $K_{1}$, since $K(V)+L$ is not closed, there exists a normalized sequence $\left(y_{n}\right)$ in $K(V)$ with dist $\left(y_{n}, L\right) \underset{n}{\longrightarrow} 0$. If $\left(y_{n}\right)$ has a subsequence weakly convergent to some $y \in Y$, since $y \in K(V)$, we may choose a sequence $\left(u_{n}\right) \subset L$ so that $\left\|u_{n}-y_{n}\right\| \underset{n}{\longrightarrow} 0$, so $u_{n} \underset{n}{\stackrel{w}{n}} y \in L$, hence $y=0$. Therefore, [4, Theorem 1.5.6] implies that $\left(y_{n}\right)$ contains a basic subsequence, and taking a bounded sequence $\left(v_{n}\right) \subset V$ such that $y_{n}=K\left(v_{n}\right)$ and passing to a subsequence if necessary, we may assume that both $\left(y_{n}\right)$ and $\left(v_{n}\right)$ are basic sequences.

Since the sequence $\left(v_{n}\right)$ is basic and $\inf _{n}\left\|v_{n}\right\|>0$, there exists a bounded sequence $\left(f_{n}\right) \subset X^{*}$ such that $\left\langle f_{i}, v_{j}\right\rangle=\delta_{i j}$. But dist $\left(y_{n}, L\right) \underset{n}{\longrightarrow}$, so we can pick a sequence $\left(z_{n}\right) \subset L$ and a subsequence $\left(y_{k_{n}}\right)$ of $\left(y_{n}\right)$ so that $\sum_{n=1}^{n}\left\|y_{k_{n}}-z_{n}\right\|<\infty$. Hence, the expression

$$
K_{1}(x):=\sum_{n=1}^{\infty}\left\langle f_{k_{n}}, x\right\rangle\left(z_{n}-y_{k_{n}}\right)
$$

defines a compact operator $K_{1} \in \mathscr{L}(X, Y)$ that satisfies $\left(K+K_{1}\right)\left(v_{k_{n}}\right)=z_{n}$. Since $\left.\left(K+K_{1}\right)\right|_{V}$ is upper semi-Fredholm and $z_{n} \in\left(K+K_{1}\right)(V) \cap L$ for every $n,(K+$ $\left.K_{1}\right)(V) \cap L$ is infinite dimensional, as we wanted to prove. 
Since all known examples of subprojective spaces are strongly subprojective, the following result implies that, in most of the cases, Theorem 2.6 is not a consequence of assertion (1) in the introduction.

Proposition 2.7. Suppose that $Y$ is subprojective and $\Phi_{+}(X, Y)$ is not empty. Then $X$ is subprojective.

Proof. It is enough to observe that $\Phi_{+}(X, Y) \neq \emptyset$ implies that a finite codimensional closed subspace of $X$ is isomorphic to a subspace of $Y$.

\section{Operators into strongly superprojective spaces}

Superprojectivity is the dual notion to subprojectivity. A Banach space $X$ is said to be superprojective if every infinite codimensional closed subspace $H$ of $X$ is contained in an infinite codimensional complemented subspace $E$ of $X$.

Definition 3.1. A Banach space $X$ is said to be strongly superprojective if every infinite codimensional closed subspace $H$ of $X$ is contained in an infinite codimensional closed subspace $E$ isomorphic to $X$ and complemented in $X$.

The proof of the following result is similar to that of Remark 2.2.

Remark 3.2. If the complement of the subspace $E$ in the definition of superprojective space can be taken isomorphic to its square, then $X$ is strongly superprojective.

Some examples of strongly superprojective Banach spaces are obtained through duality:

Proposition 3.3. Let $X$ be a reflexive Banach space. Then $X$ is strongly subprojective if and only if $X^{*}$ is strongly superprojective.

Proof. Assume $X$ is a reflexive strongly subprojective space and let $M$ be an infinite codimensional closed subspace of $X^{*}$. Thus, as $M^{\perp}$ is an infinite dimensional subspace of $X$, it contains an infinite dimensional complemented subspace $N$ with $X / N \simeq X$. Hence $N^{\perp}$ is an infinite codimensional complemented subspace of $X^{*}$ isomorphic to $X^{*}$ that contains $M$. Therefore, $X^{*}$ is strongly superprojective.

The proof of the converse implication is similar.

Observe that Proposition 3.3 is also true for superprojective and subprojective spaces.

In the following result, we list some examples of strongly superprojective spaces. Given $1<p<\infty, p^{*}$ denotes the only real number satisfying $1 / p+1 / p^{*}=1$.

Proposition 3.4. The following Banach spaces are strongly superprojective:

(1) The sequence spaces $\ell_{p}$ for $1<p<\infty$ and $c_{0}$.

(2) The dual $J^{*}$ of James' space.

(3) The dual spaces $d(w, p)^{*}$ of $d(w, p)$ for $1<p<\infty$ and $w=\left(w_{n}\right)$ a nonincreasing null sequence with $\sum_{n=1}^{\infty} w_{n}$ divergent. This applies to $\ell_{p, q}^{*}$ for $1<p, q<\infty$.

(4) The dual spaces $B_{p}^{*}$ of Baernstein's spaces for $1<p<\infty$.

(5) The dual $T^{*}$ of Tsirelson's space.

(6) The function spaces $L_{p}(0,1)$ for $1<p \leq 2$.

(7) The function spaces $L_{p}(0, \infty)+L_{2}(0, \infty)$ for $2 \leq p<\infty$. 
(8) The dual spaces $\Lambda_{W, p}(0,1)^{*}, L_{p, q}(0, \infty)^{*}$ and $L_{p, q}(0,1)^{*}$ for $2<p<\infty$ and $1<q<\infty$.

(9) The spaces of continuous functions $C(K)$, with $K$ a scattered compact.

(10) Quotients of the previous examples.

Proof. (1) The result for $\ell_{p}$ follows from Propositions 2.4 and 3.3 and, by Remark 2.3, the result for $c_{0}$ is a special case of (9).

(2) Although $J$ is non-reflexive, since $J \simeq J^{* *}$ and $\operatorname{dim} J^{* *} / J=1$, the arguments in the proof of Proposition 3.3 allow us to show that $J$ strongly subprojective implies $J^{*}$ strongly superprojective.

(3) to (8) In these cases we consider dual spaces of reflexive strongly subprojective spaces (see Proposition 2.4); therefore they are strongly superprojective by Proposition 3.3. Note that

- $d(w, p)$ is reflexive if and only if $1<p<\infty$ [18, page 178];

- $B_{p}$ is reflexive for $1<p<\infty$ [7, Theorem 0.15];

- Tsirelson's space $T$ is reflexive [18, Theorem 1.c.12] and [7, Theorem I.8];

- for $2 \leq p<\infty, L_{p}(0, \infty)+L_{2}(0, \infty)$ is the dual of $L_{p^{*}}(0, \infty) \cap L_{2}(0, \infty)$ and these spaces are reflexive [8, Theorem 3.1];

- the spaces $\Lambda_{W, p}(0,1)$ and $L_{p, q}(0, \infty)$ are reflexive for $1<p, q<\infty[10$, p. 406].

(9) Let $K$ be a scattered compact and let $M$ be a closed infinite codimensional subspace $M$ of $C(K)$. By [20, Theorem 4.2], $C(K) / M$ has a quotient isomorphic to $c_{0}$ or to $\ell_{2}$. In other words, $C(K)$ has a closed subspace $A$ with $M \subset A$ such that $C(K) / A$ is isomorphic to $c_{0}$ or to $\ell_{2}$. But $K$ is scattered, so $C(K)^{*}$ has no copy of $\ell_{2}$ because $C(K)^{*} \equiv \ell_{1}(K)$; therefore, $C(K) / A$ must be isomorphic to $c_{0}$.

Consider the quotient operator $Q_{A}: C(K) \longrightarrow C(K) / A$. Since $C(K)$ has the Pełczyński property, there exists a subspace $F$ of $C(K)$ isomorphic to $c_{0}$ such that $\left.Q_{A}\right|_{F}$ is an isomorphism. Observe that $Q_{A}(F)$ is complemented in $C(K) / A \simeq c_{0}$. So we can write $C(K) / A=Q_{A}(F) \oplus N$ for some closed subspace $N$. Hence $C(K)=$ $F \oplus Q_{A}^{-1}(N)$. We have proved that $M$ is contained in a complemented infinite codimensional subspace. Thus $C(K)$ is superprojective. Since $F$ is isomorphic to $c_{0}$ we have $F \simeq F \times F$, so Remark 3.2 shows that $C(K)$ is strongly superprojective.

(10) It is enough to prove that quotients of superprojective spaces are superprojective. Let $M$ be a closed subspace of $X$ and let $Q_{M}: X \longrightarrow X / M$ be the quotient map. Given an infinite codimensional closed subspace $A$ of $X / M, Q_{M}^{-1}(A)$ is closed an infinite codimensional in $X$ and $A \simeq Q_{M}^{-1}(A) / M$. Moreover, if $B$ is an infinite codimensional complemented subspace of $X$ containing $Q_{M}^{-1}(A)$, then $Q_{M}(B)$ is an infinite codimensional complemented subspace of $X / M$ containing $A$.

The next two results will be needed later.

Lemma 3.5. Let $K \in \mathscr{L}(X, Y)$ be an operator and $Y_{0}$ be a closed subspace of $Y$ such that $Q_{Y_{0}} K$ is surjective. If $E$ is a closed subspace of $X$ such that $K^{-1}\left(Y_{0}\right) \subset E$, then $Y$ contains a closed subspace $F$ such that $Y_{0} \subset F$ and $E=K^{-1}(F)$. Moreover, if $E$ is infinite codimensional in $X$ then $F$ is infinite codimensional in $Y$.

Proof. Consider the surjective isomorphism $U: X / N\left(Q_{Y_{0}} K\right) \longrightarrow Y / Y_{0}$ induced by $Q_{Y_{0}} K$.

Let $E$ be any closed subspace of $X$ such that $E \supset K^{-1}\left(Y_{0}\right)$. The desired subspace $F$ is $Q_{Y_{0}}^{-1} U Q_{K^{-1}\left(Y_{0}\right)}(E)$. Indeed, the facts that $F$ is closed, $F$ contains $Y_{0}$ and $E=$ 
$K^{-1}(F)$ are straightforward. Moreover, if $E$ is infinite codimensional in $X$, then $Q_{Y_{0}}^{-1} U Q_{K^{-1}\left(Y_{0}\right)}(E)$ is infinite codimensional in $Y$.

Proposition 3.6. Let $Y$ be a strongly superprojective space. Then every quotient of $Y$ by a finite dimensional subspace has a quotient isomorphic to $Y$. Therefore, $\Phi_{-}(X, Y)$ is not empty if and only if $X$ has a quotient isomorphic to $Y$.

Proof. Let $Z$ be a finite dimensional subspace of $Y$. As $Y$ is strongly superprojective, there is a closed infinite dimensional subspace $Y_{0}$ of $Y$ such that $Y / Y_{0} \simeq Y$. Let $F$ be any subspace of $Y_{0}$ with $\operatorname{dim} F=\operatorname{dim} Z$. Then

$$
\frac{Y / F}{Y_{0} / F} \simeq \frac{Y}{Y_{0}} \simeq Y
$$

and as $Y / Z \simeq Y / F$, the first assertion follows easily.

For the second assertion, the 'if' part is trivial. For the reverse, given $T \in$ $\Phi_{-}(X, Y)$, there exists a finite dimensional subspace $N$ of $Y$ such that

$$
\frac{Y}{N} \simeq R(T) \simeq \frac{X}{N(T)}
$$

thus, an application of the first assertion finishes the proof.

Next theorem is the main result of this section.

Theorem 3.7. Let $Y$ be a strongly superprojective space and let $X$ be a Banach space such that $\Phi_{-}(X, Y) \neq \emptyset$. Then $P \Phi_{-}(X, Y)=\mathscr{S} \mathscr{C}(X, Y)$.

Proof. It is enough to show that, given $K \in \mathscr{L}(X, Y) \backslash \mathscr{S} \mathscr{C}(X, Y)$, there exists $T \in \Phi_{-}(X, Y)$ such that $T+K \notin \Phi_{-}$. In order to do that, let $K: X \longrightarrow Y$ be a non-strictly cosingular operator. Thus there exists a closed subspace $Y_{0} \subset Y$ with $\operatorname{dim} Y / Y_{0}=\infty$ such that $Q_{Y_{0}} K$ is surjective, where $Q_{Y_{0}}$ is the quotient operator onto $Y / Y_{0}$. Obviously, $R(K)+Y_{0}=Y$.

Since $Y$ is strongly superprojective, the space $Y_{0}$ can be assumed to be isomorphic to $Y$ and complemented in $Y$. Thus $Y=Y_{0} \oplus N$ with $\operatorname{dim} N=\infty$.

Let $P: Y \longrightarrow Y$ be the projection with $N(P)=Y_{0}$ and $R(P)=N$. Thus

$$
\begin{aligned}
& R(P K)=P\left(R(K)+Y_{0}\right)=N, \\
& N(P K)=K^{-1}\left(Y_{0}\right) .
\end{aligned}
$$

As $\Phi_{-}(X, Y) \neq \emptyset$, Proposition 3.6 provides a closed subspace $M$ of $X$ such that $X / M \simeq Y$. Hence, as $Y \simeq Y_{0}$, there exists $S \in \mathscr{L}(X, Y)$ such that $N(S)=M$ and $R(S)=Y_{0}$.

Taking into account the relative positions of $K^{-1}\left(Y_{0}\right)$ and $M$ in $X$, three cases occur:

(a) $K^{-1}\left(Y_{0}\right)+M$ is closed and finite codimensional in $X$,

(b) $\overline{K^{-1}\left(Y_{0}\right)+M}$ is infinite codimensional in $X$,

(c) $\overline{K^{-1}\left(Y_{0}\right)+M}$ is finite codimensional in $X$ but $K^{-1}\left(Y_{0}\right)+M$ is not closed.

(a) Let $M_{1}$ be a subspace of $X$ containing $M$ such that $\operatorname{dim} M_{1} / M<\infty$ and $K^{-1}\left(Y_{0}\right)+M_{1}=X$. Since $X / M$ is superprojective, by Proposition 3.6, there exists a closed subspace $M_{2}$ containing $M_{1}$ such that $X / M_{2} \simeq Y$. Thus we can assume $K^{-1}\left(Y_{0}\right)+M=X$. 
Let $T:=S-P K$. Observe that $R(T)=Y$. Indeed, given $y \in Y$, decompose $y=y_{0}+y_{1}$ with $y_{0} \in Y_{0}$ and $y_{1} \in N$.

We will show that

$$
\begin{aligned}
& y_{0}=S\left(x_{0}\right) \text { for some } x_{0} \in K^{-1}\left(Y_{0}\right), \\
& y_{1}=P K\left(x_{1}\right) \text { for some } x_{1} \in M .
\end{aligned}
$$

In order to prove (1), take $x \in X$ such that $y_{0}=S(x)$ and consider any decomposition $x=x_{0}+x_{0}^{\prime}$ with $x_{0} \in K^{-1}\left(Y_{0}\right)$ and $x_{0}^{\prime} \in M$. Thus $x_{0}^{\prime} \in N(S)$, hence $y_{0}=S\left(x_{0}\right)$.

For (2), as $N=R(P K)$, there exists $x \in X$ such that $y_{1}=P K(x)$. Take any decomposition $x=x_{1}+x_{1}^{\prime}$ with $x_{1} \in M$ and $x_{1}^{\prime} \in K^{-1}\left(Y_{0}\right)$. Since $K\left(x_{1}^{\prime}\right) \in Y_{0}=$ $N(P)$, it follows that $y_{1}=P K\left(x_{1}\right)$, and (2) is proved.

Finally, formulas (1) and (2) yield that $x_{0} \in N(P K)$ and $x_{1} \in N(S)$, hence $y=$ $(S-P K)\left(x_{0}-x_{1}\right)$. We have just proved that $T$ is surjective, hence, $T \in \Phi_{-}(X, Y)$.

However, $R(T+K)=R\left(S+\left(I_{X}-P\right) K\right) \subset Y_{0}$, so $T+K \notin \Phi_{-}(X, Y)$.

(b) Assume $\overline{K^{-1}\left(Y_{0}\right)+M}$ is infinite codimensional. Thus $\left(\overline{K^{-1}\left(Y_{0}\right)+M}\right) / M$ is an infinite codimensional subspace of $X / M$. But $X / M$ is isomorphic to $Y$, so it is strongly superprojective, hence there exists a closed infinite codimensional subspace $X_{1}$ of $X$ such that $\overline{K^{-1}\left(Y_{0}\right)+M} \subset X_{1}, X_{1} / M \simeq Y$ and

$$
\frac{X}{M}=\frac{X_{1}}{M} \oplus \frac{E}{M}
$$

for some subspace $M \subset E \subset X$ with $\operatorname{dim} E / M=\infty$. Lemma 3.5 provides a closed subspace $Y_{1}$ of $Y$ such that $\operatorname{dim} Y / Y_{1}=\infty, Y_{0} \subset Y_{1}$ and $X_{1}=K^{-1}\left(Y_{1}\right)$. Thus

$$
K^{-1}\left(Y_{1}\right)+E=X_{1}+E=X \text {. }
$$

Moreover,

$$
X / E \simeq \frac{X / M}{E / M} \simeq X_{1} / M \simeq Y .
$$

Therefore, using again that $Y$ is strongly superprojective, there exists a complemented infinite codimensional subspace $Y_{2}$ of $Y$ such that $Y_{1} \subset Y_{2} \subset Y$ and $Y_{2} \simeq Y$.

Obviously, $Q_{Y_{2}} K$ is surjective, $X / E \simeq Y$ and $K^{-1}\left(Y_{2}\right)+E=X$ so we are in the conditions of case (a) (using an operator $S_{1} \in \mathscr{L}(X, Y)$ with $N\left(S_{1}\right)=E$ and $R\left(S_{1}\right)=Y_{2}$, instead of $\left.S\right)$.

(c) As in the case (a), we can assume that $K^{-1}\left(Y_{0}\right)+M$ is dense but not closed in $X$. We will find a compact operator $K_{1} \in \mathscr{L}(X, Y)$ such that $\overline{\left(K+K_{1}\right)^{-1}\left(Y_{0}\right)+M}$ is infinite codimensional in $X$. Once $K_{1}$ has been found, as $\left.R\left(Q_{Y_{0}}\left(K+K_{1}\right)\right)\right)$ is finite codimensional in $Y / Y_{0}$, there exists a finite rank operator $K_{2} \in \mathscr{L}(X, Y)$ such that $Q_{Y_{0}}\left(K+K_{1}+K_{2}\right)$ is surjective and $\overline{\left(K+K_{1}+K_{2}\right)^{-1}\left(Y_{0}\right)+M}$ is infinite codimensional in $X$ yet. Hence, applying the argument of (b), we get that $K+K_{1}+$ $K_{2} \notin P \Phi_{-}(X, Y)$, and as $K_{1}+K_{2}$ is compact, we can conclude that $K \notin P \Phi_{-}(X, Y)$.

In order to find $K_{1}$, since $K^{-1}\left(Y_{0}\right)+M$ is not closed, it follows $K^{-1}\left(Y_{0}\right)^{\perp}+M^{\perp}$ is not closed either; but $X=\overline{K^{-1}\left(Y_{0}\right)+M}$, so

$$
\{0\}=K^{-1}\left(Y_{0}\right)^{\perp} \cap M^{\perp} .
$$

Thus, we may take a normalized sequence $\left(f_{n}\right) \subset K^{-1}\left(Y_{0}\right)^{\perp}$ such that $\operatorname{dist}\left(f_{n}, M^{\perp}\right)<$ $1 / 2^{n}$. Take also a sequence $\left(h_{n}\right)$ in $M^{\perp}$ so that $\left\|f_{n}-h_{n}\right\|<1 / 2^{n}$. 
Note that $\left(f_{n}\right)$ does not have any convergent subsequence; otherwise, if $f_{k_{n}} \underset{n}{\longrightarrow} f$, then $h_{k_{n}} \underset{n}{\longrightarrow} f$ too, so $f=0$ because of (3), a contradiction.

Let $f$ be a weak* cluster point of $\left(f_{n}\right)$. As both subspaces $K^{-1}\left(Y_{0}\right)^{\perp}$ and $M^{\perp}$ are weak* closed, (3) yields that $f=0$. Thus, by [13, Lemma 3.1.19], there is a bounded sequence $\left(x_{n}\right)$ in $X$ and a basic subsequence $\left(f_{k_{n}}\right)$ of $\left(f_{n}\right)$ such that $\left\langle f_{k_{n}}, x_{m}\right\rangle=\delta_{n m}$.

As $K^{-1}\left(Y_{0}\right)^{\perp}=K^{*}\left(Y_{0}^{\perp}\right)$, we may pick a sequence $\left(g_{n}\right)$ in $Y_{0}^{\perp}$ such that $K^{*}\left(g_{n}\right)=$ $f_{k_{n}}$; note that $\left(g_{n}\right)$ is bounded because $\left.K^{*}\right|_{Y_{0}^{\perp}}$ is an isomorphism. Let $y_{n}:=K\left(x_{n}\right)$. Obviously,

$$
\left\langle g_{i}, y_{j}\right\rangle=\left\langle K^{*}\left(g_{i}\right), x_{j}\right\rangle=\delta_{i j} .
$$

Consider the compact operator $K_{1}: X \longrightarrow Y$ given by the expression

$$
K_{1}(x):=\sum_{n=1}^{\infty}\left\langle h_{k_{n}}-f_{k_{n}}, x\right\rangle y_{n} .
$$

Its conjugate operator is given by $K_{1}^{*}(g)=\sum_{n=1}^{\infty}\left\langle g, y_{n}\right\rangle\left(h_{k_{n}}-f_{k_{n}}\right)$. Thus $\left(K^{*}+\right.$ $\left.K_{1}^{*}\right)\left(g_{n}\right)=h_{k_{n}} \in\left(K^{*}+K_{1}^{*}\right)\left(Y_{0}^{\perp}\right) \cap M^{\perp}$ for all $n$, which proves that $\left(K^{*}+K_{1}^{*}\right)\left(Y_{0}^{\perp}\right) \cap$ $M^{\perp}$ is infinite dimensional. But

$$
\left(K^{*}+K_{1}^{*}\right)\left(Y_{0}^{\perp}\right) \cap M^{\perp}=\left(K+K_{1}\right)^{-1}\left(Y_{0}\right)^{\perp} \cap M^{\perp}={\overline{\left(K+K_{1}\right)^{-1}\left(Y_{0}\right)+M^{\perp}}}^{\perp}
$$

hence $\overline{\left(K+K_{1}\right)^{-1}\left(Y_{0}\right)+M}$ is an infinite codimensional subspace of $X$, as we wanted to prove. The proof is done.

Since all known examples of superprojective spaces are strongly superprojective, the following result implies that, in most of the cases, Theorem 3.7 is not a consequence of assertion (1') in the introduction.

Proposition 3.8. Assume that $X$ is superprojective and $\Phi_{-}(X, Y)$ is not empty. Then $Y$ is superprojective.

Proof. It is enough to note that $\Phi_{-}(X, Y) \neq \emptyset$ implies that a quotient of $Y$ by a finite dimensional subspace is isomorphic to a quotient of $X$.

\section{References}

[1] Aiena, P., and M. GonzÁlez: Inessential operators between Banach spaces. - Rend. Circ. Mat. Palermo (2) 68, 2002, 3-26.

[2] Aiena, P., M. González, and A. Martínez-Abejón: Operator semigroups in Banach space theory. - Boll. Unione Mat. Ital. Sez. B Artic. Ric. Mat. (8) 4, 2001, 157-205.

[3] Aiena, P., M. González, and A. Martinón: On the perturbation classes of continuous semi-Fredholm operators. - Glasgow Math. J. 45, 2003, 91-95.

[4] Albiac, F., and N. Kalton: Topics in Banach space theory. - Springer, New York, 2006.

[5] Caradus, S., W. Pfaffenberger, and B. Yood: Calkin algebras and algebras of operators in Banach spaces. - Lecture Notes in Pure and Appl. Math., M. Dekker, New York, 1974.

[6] Carothers, N. L., and S. J. Dilworth: Subspaces of $L_{p, q}$ - Proc. Amer. Math. Soc. 104, 1988, 537-545.

[7] Casazza, P., and T. J. Shura: Tsirelson's space. - Lecture Notes in Math. 1363, Springer, 1989.

[8] Dilworth, S. J.: A scale of linear spaces related to the $L_{p}$ scale. - Illinois J. Math. 34, 1990, $140-158$. 
[9] Fetter, H., and B. Gamboa De Buen: The James forest. - London Math. Soc. Lecture Note Ser. 236, Cambridge Univ. Press, Cambridge, 1997.

[10] Figiel, T., W. B. Johnson, and L. Tzafriri: On Banach lattices and spaces having local unconditional structure, with application to Lorentz function spaces. - J. Approx. Theory 13, $1975,395-412$.

[11] Gohberg, I. C., A. S. Markus, and I. A. Feldman: Normally solvable operators and ideals associated with them. - Bul. Akad. Štiince RSS Moldoven 10:76, 1960, 51-70; English transl.: Amer. Math. Soc. Transl. (2) 61, 1967, 63-84.

[12] GonzÁlez, M.: The perturbation classes problem in Fredholm theory. - J. Funct. Anal. 200, 2003, 65-70.

[13] González, M., and A. Martínez-Abejón: Tauberian operators. - Oper. Theory Adv. Appl. 194, Birkhäuser, Basel, 2010.

[14] GonzÁlez, M., and M. Salas-Brown: Perturbation classes for semi-Fredholm operators on $L_{p}(\mu)$-spaces. - J. Math. Anal. Appl. 370, 2010, 11-17.

[15] Gowers, W. T., and B. Maurey: The unconditional basic sequence problem. - J. Amer. Math. Soc. 6, 1993, 851-874.

[16] Kato, T.: Perturbation theory for nullity, deficiency and other quantities of linear operators. - J. Anal. Math. 6, 1958, 261-322.

[17] Lebow, A., and M. Schechter: Semigroups of operators and measures of noncompactness. - J. Funct. Anal. 7, 1971, 1-26.

[18] Lindenstrauss, J., and L. Tzafriri: Classical Banach spaces I. Sequence spaces. - Springer, Berlin, 1977.

[19] Lotz, H. P., N. T. Peck, and H. Porta: Semi-embeddings of Banach spaces. - Proc. Edinburgh Math. Soc. 22, 1979, 233-155.

[20] Música, J.: Separable quotients of Banach spaces. - Rev. Mat. Complut. 10, 1997, 299-330.

[21] PeeczyŃski, A.: On strictly singular and strictly cosingular operators II. Strictly singular and strictly cosingular operators in $L(\nu)$-spaces. - Bull. Acad. Polon. Sci. 13, 1965, 37-41.

[22] Pietsch, A.: Operator ideals. - North-Holland, Amsterdam, 1980.

[23] Tylli, H.-O.: Lifting non-topological divisors of zero modulo the compact operators. - J. Funct. Anal. 125, 1994, 389-415.

[24] VladimirskiI, J. I.: Strictly cosingular operators. - Soviet Math. Dokl. 8, 1967, 739-740.

[25] Weis, L.: On perturbation of Fredholm operators in $L_{p}(\mu)$-spaces. - Proc. Amer. Math. Soc. 67, 1977, 287-292.

[26] Whitley, R. J.: Strictly singular operators and their conjugates. - Trans. Amer. Math. Soc. $113,1964,252-261$.

Received 16 April 2010 